\title{
ÍNDICES DE CRESCIMENTO ESTATURAL E ESTADO NUTRICIONAL DE ESCOLARES DE 6 A 14 ANOS
}

\author{
Glauber Carvalho Nobre, Universidade Federal do Rio Grande do Sul - UFRGS, \\ Ceará-Brasil \\ Karla Sibelle Camps de Lima, Universidade Regional do Cariri-URCA, Crato, Ceará \\ - Brasil \\ Antônia Deliane Gomes da Silva, Universidade Regional do Cariri - URCA, Crato, \\ Ceará-Brasil \\ Anastácio Neco de Souza Filho, Universidade Regional do Cariri - URCA, Crato, \\ Ceará-Brasil \\ Rubens Vinícius Letieri, Faculdade Católica Rainha do Sertão - FCRS, Quexidá, Ceará \\ - Brasil \\ Paulo Felipe Ribeiro Bandeira, Universidade Federal do Rio Grande do Sul-UFRGS, \\ Ceará - Brasil
}

\section{RESUMO}

O objetivo desse estudo foi investigar os índices de crescimento estatural e estado nutricional de escolares da rede municipal de ensino de Acopiara, no estado do Ceará. Participaram do estudo 622 escolares, dos quais 323 do sexo masculino e 299 do feminino com faixa etária entre 06 e 14 anos matriculados em duas escolas públicas do referido município. Para a avaliação do crescimento físico e estado nutricional este estudo adotou as variáveis massa corporal, estatura e os índices estatura para idade e IMC para idade de acordo com a World Health Organization (2007). Em relação à estatura para idade, percebeu-se conforme o esperado, que a maioria dos indivíduos independente da idade indicou estatura adequada. Entretanto, verificou-se um percentual elevado de indivíduos, sobretudo no grupo masculino, com baixa estatura para idade. Quanto ao IMC para idade verificou-se novamente conforme o esperado, que a maioria dos indivíduos independente da idade indicou IMC adequado. Porém, verificou-se um percentual significativamente elevado de indivíduos, tanto no grupo masculino como feminino com classificação do estado nutricional alusivo à magreza e magreza acentuada. Estes resultados podem indicar que estes indivíduos possam estar sofrendo uma carência nutricional podendo levá-los a apresentar prejuízo no desenvolvimento psicomotor além de menor aproveitamento escolar.

Palavras-Chave: Estatura; Peso corporal; Crescimento físico; Estudantes.

\section{GROWTH AND NUTRITIONAL STATUS OF SCHOOL OF 6 TO 14 YEARS}

\begin{abstract}
The aim of this study was to investigate the rate of height growth and nutritional status of schoolchildren in the municipal education Acopiara-Ce. Participants were 622 students, including 323 males and 299 females aged from 06 to 14 years enrolled in two public schools in the municipality. For assessing the physical growth and nutritional status this study adopted the variables body mass, height and their height for age and BMI for age according to the World Health Organization (2007). In relation to height

Conexões: revista da Faculdade de Educação Física da UNICAMP, Campinas, v. 12, n. 1, p. 126-141, jan./mar. 2014. ISSN: 1983-9030
\end{abstract}


for age, it was realized as expected, that most individuals regardless of age indicated proper height. However, there was a high percentage of individuals, particularly males, with stunting. As the BMI for age verified again as expected that most individuals regardless of age appropriate BMI indicated. However, there was a significantly higher percentage of individuals in both the male and female with the nutritional status illustrating the sharp thinness and thinness. These results may indicate that these individuals may be suffering a nutritional deficiency can cause them to experience impairments in psychomotor development in addition to academic underachievement.

Key-Words: Height; Weight; Physical growth; Students.

\section{LAS TASAS DE CRECIMIENTO EN ALTURA Y EL ESTADO NUTRICIONAL DE LOS ESCOLARES DE EDADES 06 A 14 AÑOS}

\section{RESUMEN}

El objetivo de este estudio fue investigar las tasas de crecimiento en altura y el estado nutricional de ninõs escolares de las escuelas de la municipalidad de Acopiara -Ce . En el estudio participaron 622 estudiantes, incluyendo 323 hombres y 299 mujeres de 06 a 14 matriculados en dos escuelas públicas de dicha municipalidad. Para evaluar el crecimiento físico y el estado nutricional de este estudio se adoptó las variables peso , altura y los índices de baja estatura para la edad y el IMC para la edad, según la Organización Mundial de la Salud (2007). En relación con la estatura para la edad, se mantuvo como se esperaba, la mayoría de las personas independientemente de su edad indicaron altura adecuada. Sin embargo, hubo un alto porcentaje de las personas, en particular los varones, con retraso en el crecimiento . Como se observó, el índice de masa corporal para la edad, de nuevo como era de esperar, la mayoría de las personas independientemente de la edad indicada IMC adecuado. Sin embargo, hubo un porcentaje significativamente mayor de individuos, hombres y mujeres para determinar el estado nutricional alusiva a la delgadez y la delgadez severa. Estos resultados podrían indicar que estas personas pueden estar sufriendo de una deficiencia nutricional y puede hacer que se experimentan alteraciones el desarrollo psicomotor, además de menor nivel educativo.

Palabras-Claves: Altura; Peso; Crecimiento físico; Estudiantes. 


\section{INTRODUÇÃO}

O crescimento físico e o estado nutricional são fatores que podem indicar aspectos de saúde de uma população. ${ }^{1} \mathrm{O}$ crescimento físico está diretamente ligado ao estado nutricional, sendo que uma alimentação adequada é condição fundamental para estas duas variáveis. É importante que se tenha um equilíbrio entre o consumo alimentar e as necessidades metabólicas diárias específicas do organismo, pois uma deficiência na quantidade ou qualidade de nutrientes consumidos no dia a dia vai constituir uma das causas imediatas mais significativas dos problemas de saúde e nutrição de uma população. ${ }^{2}$

Para uma boa assistência a saúde da criança e do adolescente é indispensável que se tenha um controle do seu crescimento, através de uma comparação da sua massa corporal, estatura e quantidade de gordura corporal e considerando também as variáveis ambientais que influenciam significativamente estes parâmetros. ${ }^{3}$ Em diversos países o crescimento físico é um dos mais importantes indicadores do nível de saúde da população e a sua avaliação na infância e adolescência permite detectar precocemente, individualmente e comparando com um grupo, problemas de subnutrição, baixa estatura para a idade e obesidade. Contudo essas análises devem levar em conta os aspectos biológicos e sociais do público alvo. ${ }^{4}$ A avaliação do estado nutricional também é um importante indicador do nível de saúde de crianças e adolescentes visto que crianças que apresentam obesidade têm maior probabilidade de apresentar alguns problemas de saúde como a elevação da pressão sanguínea, dislipidemia, maior prevalência de fatores associados com a resistência insulínica e ao diabetes tipo $I$ II, $^{5}$ já as que têm um quadro de desnutrição apresentam um maior nível de doenças infecciosas, prejuízo para o desenvolvimento psicomotor, menor aproveitamento escolar e menor capacidade produtiva na idade adulta. ${ }^{6}$

Estudos realizados nas mais diferentes regiões do Brasil $^{7-9}$ têm revelado prevalência significativa de crianças e adolescentes em risco de obesidade e obesidade e declínio na ocorrência de desnutrição. Na região Sul e Sudeste os resultados revelaram a prevalência de risco de obesidade e obesidade grave variou entre 4,7\% a 12,7\%. Esses

Conexões: revista da Faculdade de Educação Física da UNICAMP, Campinas, v. 12, n. 1, p. 126-141, jan./mar. 2014. ISSN: 1983-9030 
níveis podem estar relacionados a uma possível modernização nas condições de vida, nessa região, frequentemente associadas à supernutrição e sedentarismo. ${ }^{7}$

Já para a região Nordeste percebeu-se que a prevalência de crianças e adolescentes com sobrepeso e obesidade é menor, sendo que a maior parte dos que ainda apresentaram obesidade foram crianças e adolescentes do sexo feminino. ${ }^{9}$ Em outro estudo realizado também na região nordeste do Brasil $^{10}$ constatou-se os índices de sobrepeso mais frequente entre as crianças do que entre os adolescentes, sendo maior também no sexo masculino e a taxa de obesidade foi de $8,5 \%$, indicando que os níveis de obesidade mostraram-se também mais prevalentes entre as crianças do que entre os adolescentes. ${ }^{10}$ Nobre et $\mathrm{al}^{11}$ analisou o perfil de crescimento físico e estado nutricional de escolares oriundos de um município do localizado no interior do nordeste brasileiro, e confirmou uma alta prevalência de magreza e risco de obesidade.

Entretanto as pesquisas sobre crescimento físico e estado nutricional ainda concentram dados de crianças em regiões centrais do nordeste, tornando-se imprescindível a realização de estudos em municípios do interior desta região. Diante das informações acima expostas e da carência de informações com relação ao interior do Nordeste surge o seguinte questionamento: Quais são os índices de crescimento estatural e estado nutricional de escolares de 6 a 14 anos oriundos de um município situado na região centro-sul do estado do Ceará?

\section{MATERIAL E MÉTODOS}

\section{CARACTERIZAÇÃO DO ESTUDO}

O presente estudo caracteriza-se como descritivo transversal. ${ }^{12}$

\section{PARTICIPANTES DO ESTUDO}

Os participantes do estudo foram escolares de ambos os sexos com faixa etária entre 06 e 14 anos, estudantes do $1^{\circ}$ ao $9^{\circ}$ ano do ensino fundamental, totalizando 622 participantes, dos quais 323 do sexo masculino e 299 do feminino (TABELA 1), matriculados em duas escolas públicas do município de Acopiara/CE.

Conexões: revista da Faculdade de Educação Física da UNICAMP, Campinas, v. 12, n. 1, p. 126-141, jan./mar. 2014. ISSN: 1983-9030 
Tabela 1 - Distribuição da amostra estratificada por idade e sexo

\begin{tabular}{cccc}
\hline Idade & Meninos & Meninas & Total \\
\hline $6 \pm$ & 37 & 28 & 65 \\
$7 \pm$ & 36 & 33 & 69 \\
$8 \pm$ & 38 & 30 & 68 \\
$9 \pm$ & 21 & 38 & 59 \\
$10 \pm$ & 39 & 33 & 72 \\
$11 \pm$ & 43 & 30 & 73 \\
$12 \pm$ & 37 & 44 & 81 \\
$13 \pm$ & 39 & 38 & 77 \\
$14 \pm$ & 33 & 26 & 59 \\
\hline
\end{tabular}

\section{CARACTERIZANDO O CONTEXTO DA PESQUISA}

O estudo se concentrou na sede de Acopiara, o município se encontra em $70^{\circ}$ lugar no ranking do Índice FIRJAN de Desenvolvimento Municipal que é um estudo anual do Sistema Federação das Indústrias do Estado do Rio de Janeiro - FIRJAN que acompanha o desenvolvimento de todos os 5.564 municípios brasileiros em três áreas: emprego \& renda, educação e saúde. É feito exclusivamente, com base em estatísticas públicas oficiais, disponibilizadas pelos ministérios do trabalho, educação e saúde. Acopiara ocupa uma classificação mediana nos índices de educação e saúde, representando uma tendência de evolução nas referidas categorias em comparação com o estudo realizado no ano anterior. ${ }^{13}$

\section{INSTRUMENTOS}

Foi utilizado o Formulário para avaliação clínica e psicossocial do Programa saúde na escola - PSE, do qual foram coletados os dados de massa corporal e estatura. 


\section{PROCEDIMENTOS}

Para a realização do estudo foram utilizados dados de peso e estatura provenientes do Programa Saúde na Escola (PSE), que é um programa do Ministério da Saúde e do Ministério da Educação que tem perspectiva de ampliar ações especificas de saúde para alunos da rede pública de ensino. Dessa forma, foi feito o contato a coordenação do PSE, assim como, com a diretoria e coordenação de algumas escolas da sede (zona urbana) do município, para permissão de utilização dos dados do mesmo (assinatura do termo de fiel depositário).

Este estudo adotou as variáveis: massa corporal (mc), estatura (est) e o indicador de Estatura para Idade ${ }^{14}$ no qual considera os seguintes valores críticos: estatura menor que o escore z -3: Muito baixa estatura para a idade; estatura entre o escore z -3 e -2 : baixa estatura para a idade; estatura maior ou igual ao escore z -2: estatura adequada para a idade. ${ }^{14} \mathrm{O}$ estado nutricional será avaliado por meio do Índice de Massa Corporal () normatizado pela Idade onde os valores de corte para classificação obedeceram aos seguintes critérios: Magreza acentuada: IMC menor que o escore z -3; Magreza: escore $\mathrm{z}$ entre -3 e -2; IMC Normal: escore $\mathrm{z}$ entre -2 e 1 ; risco de obesidade: escore $\mathrm{z}$ entre 1 e 2; obesidade: escore $\mathrm{z}$ maior que $2 .{ }^{14}$ Para plotagem dos gráficos de crescimento estatural (estatura para idade) e estado nutricional (IMC para idade) foi utilizado o software Anthro®.

\section{ANÁLISE ESTATÍSTICA}

Para análise dos dados, aplicou-se a priori, o teste de Kolmogorov-Smirnov observando uma distribuição normal das variáveis: massa corporal, estatura e índice de massa corporal.

Posteriormente, os resultados foram analisados por meio da estatística descritiva (média, desvio padrão, frequência e percentual). Para verificar possíveis diferenças nas variáveis massa corporal, estatura e IMC entre os sexos em cada idade utilizou-se o teste " $t$ " de student para amostras independentes. Empregou-se também a análise de variância one way para verificar possíveis diferenças intra sexos nestas variáveis entre as idades. O nível de significância adotado foi de $\square<0,050$.

Conexões: revista da Faculdade de Educação Física da UNICAMP, Campinas, v. 12, n. 1, p. 126-141, jan./mar. 2014. ISSN: 1983-9030 


\section{RESULTADOS E DISCUSSÃO}

A comparação dos indicadores estatura, massa corporal e índice de massa corporal conforme com o sexo e idade são apresentados na Tabela 2 abaixo. Na mesma não se observou diferenças entre meninos e meninas nas diferentes idades em relação a estatura e também a massa corporal configurando um resultado atípico, pois no início da adolescência as meninas tendem a apresentar maior estatura e massa corporal. Quando a partir dos 14 anos os meninos ultrapassam as meninas, este resultado pode ser explicado pelo atraso na entrada do estirão do crescimento por parte das meninas. Ainda referente a Figura 1 (gráficos de dinâmica de crescimento), percebe-se que tanto meninos como meninas apresentam um crescimento linear para estatura e para peso, contudo também não apresentaram diferença nestes dois indicadores. Com relação a um grupo de mesmo sexo e idade cronológica há a possibilidade de variação na maturação, onde as crianças apresentam sua própria trajetória rumo ao crescimento, podendo ser de forma avançada, normal ou atrasada. ${ }^{15}$ Contudo os resultados encontrados no presente estudo vão ao encontro dos apresentados na cidade de Minas Gerais que também apresentou valores semelhantes com relação aos valores de massa corporal e estatura até os 14 anos, onde depois dessa idade os adolescentes ultrapassaram as moças com relação às índices mencionados anteriormente. ${ }^{16}$

Tabela 2 - Comparação dos indicadores Estatura, Massa Corporal e Índice de Massa Corporal conforme com o sexo e idade

\begin{tabular}{|c|c|c|c|c|c|c|c|c|c|}
\hline \multirow{2}{*}{ Idade } & \multicolumn{2}{|c|}{ Estatura $(\mathbf{c m})$} & \multirow[b]{2}{*}{$\begin{array}{c}\mathrm{p} \\
\text { valor }\end{array}$} & \multicolumn{2}{|c|}{ Massa Corporal (kg) } & \multicolumn{4}{|c|}{$\begin{array}{c}\text { Índice de Massa } \\
\text { Corporal }\left(\mathrm{kg} / \mathrm{m}^{2}\right) \\
\end{array}$} \\
\hline & Masculino & Feminino & & Masculino & Feminino & $\begin{array}{c}\mathrm{p} \\
\text { valor }\end{array}$ & Masculino & Feminino & $\begin{array}{c}\mathrm{p} \\
\text { valor }\end{array}$ \\
\hline 6 anos & $118,5+5,9$ & $119,6 \pm 5,5$ & 0,999 & $21,1+3,6$ & $23,8 \pm 6,6$ & 0,998 & $14,9+1,8$ & $16,0 \pm 3,7$ & 0,996 \\
\hline 7 anos & $125,2+5,1$ & $124,0 \pm 6,5$ & 0,997 & $24,0+3,1$ & $23,1 \pm 4,2$ & 0,999 & $15,3+1,6$ & $14,9 \pm 1,7$ & 0,996 \\
\hline 8 anos & $127,8+7,7$ & $129,8 \pm 6,3$ & 0,998 & $25,6+6,0$ & $28,9 \pm 8,0$ & 0,997 & $15,5+2,5$ & $16,9 \pm 3,5$ & 0,997 \\
\hline 9 anos & $131,4+7,3$ & $135,3 \pm 7,7$ & 0,983 & $27,9+5,1$ & $29,1 \pm 6,8$ & 0,987 & $16,1+2,2$ & $15,8+2,7$ & 0,995 \\
\hline $\begin{array}{c}10 \\
\text { anos }\end{array}$ & $137,5+7,4$ & $138,5 \pm 10,4$ & 0,997 & $31,6+7,6$ & $32,8 \pm 7,7$ & 0,988 & $16,5+2,5$ & $17,1 \pm 3,7$ & 0,996 \\
\hline $\begin{array}{c}11 \\
\text { anos }\end{array}$ & $143,8+7,1$ & $144,0 \pm 7,8$ & 0,997 & $35,4+6,5$ & $37,1 \pm 9,5$ & 0,999 & $17,0+2,4$ & $17,6 \pm 3,1$ & 0,999 \\
\hline $\begin{array}{c}12 \\
\text { anos }\end{array}$ & $149,5+8,1$ & $149,6 \pm 8,8$ & 0,999 & $39,7+9,1$ & $41,3 \pm 11,5$ & 0,999 & $17,7+3,4$ & $18,2 \pm 3,6$ & 0,997 \\
\hline $\begin{array}{c}13 \\
\text { anos }\end{array}$ & $156,0+10,2$ & $152,6 \pm 7,5$ & 0,912 & $44,0+10,1$ & $41,6 \pm 7,8$ & 0,994 & $17,9+3,1$ & $17,8 \pm 2,7$ & 0,999 \\
\hline $\begin{array}{c}14 \\
\text { anos }\end{array}$ & $160,3+9,5$ & $157,1 \pm 6,9$ & 0,974 & $46,5+9,3$ & $47,1 \pm 9,3$ & 0,982 & $17,9+2,2$ & $19,0 \pm 3,1$ & 0,975 \\
\hline
\end{tabular}

Conexões: revista da Faculdade de Educação Física da UNICAMP, Campinas, v. 12, n. 1, p. 126-141, jan./mar. 2014. ISSN: 1983-9030 


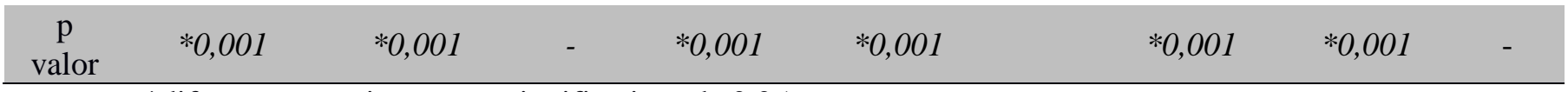

*diferenças estatiscamente significativas de 0,05
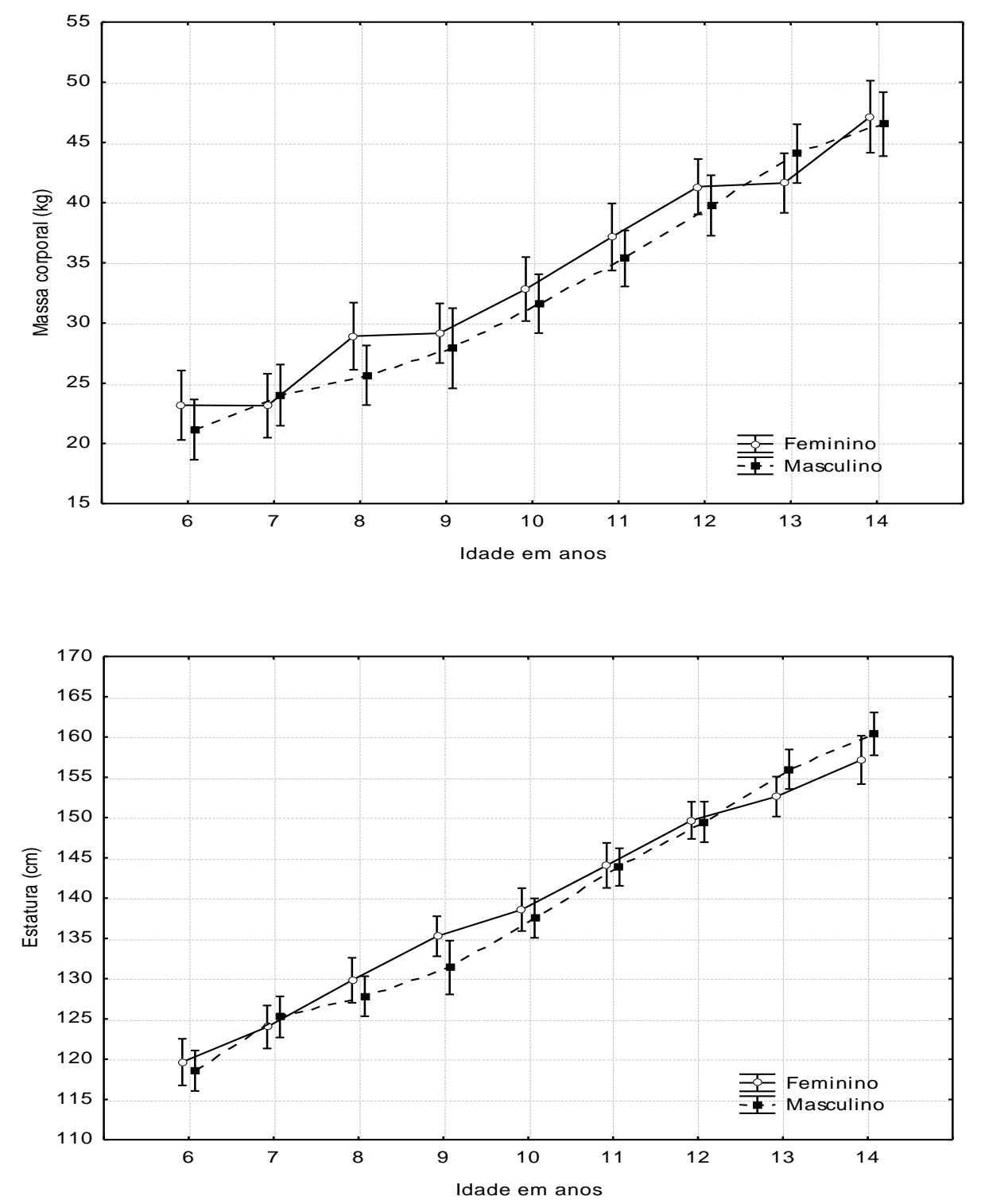

Figura 1: Dinâmica da massa corporal e estatura das crianças conforme o sexo e a idade

Ao observar a classificação da estatura para idade (TABELA 3) percebeu-se, conforme o esperado, que a maioria dos indivíduos independente da idade indicou estatura

Conexões: revista da Faculdade de Educação Física da UNICAMP, Campinas, v. 12, n. 1, p. 126-141, jan./mar. 2014. ISSN: 1983-9030 
adequada. Entretanto, verificou-se um percentual elevado de indivíduos, sobretudo no grupo masculino, com baixa estatura para idade, dentre os quais os que se encontram na faixa etária de 09, 13 e 14 anos apresentando um percentual para esta classificação de $(14,3 \%),(15,4 \%)$ e $(9,1 \%)$ respectivamente. Já no grupo feminino apenas $(10 \%)$ e $(7,9 \%)$ das adolescentes de 11 e 13 anos respectivamente indicaram baixa estatura para idade. As curvas de estatura para idade dos meninos e meninas investigados apresentaram uma concentração significativa nas áreas entre o escore z -3 e -2 que é o intervalo que determina a classificação de baixa estatura para a idade. (FIGURA 2).

Tabela 3 - Classificação da estatura para idade de acordo com o sexo

\begin{tabular}{cccc}
\hline Sexo e Idade & $\begin{array}{c}\text { Muito Baixa } \\
\text { estatura para } \\
\text { idade }\end{array}$ & $\begin{array}{c}\text { Baixa estatura } \\
\text { para idade }\end{array}$ & $\begin{array}{c}\text { Estatura adequada } \\
\text { para idade }\end{array}$ \\
\hline Masculino & - & - & $37(100)$ \\
6 anos & - & - & $36(100)$ \\
7 anos & - & $3(7,9)$ & $35(92,1)$ \\
8 anos & - & $3(14,3)$ & $18(85,7)$ \\
9 anos & - & $1(2,6)$ & $38(97,4)$ \\
10 anos & - & $2(4,7)$ & $41(95,3)$ \\
11 anos & $1(2,7)$ & $2(5,4)$ & $34(91,9)$ \\
12 anos & - & $6(15,4)$ & $33(84,6)$ \\
13 anos & - & $3(9,1)$ & $30(90,9)$ \\
14 anos & - & - & $28(100)$ \\
\hline Feminino & - & - & $32(97)$ \\
6 anos & $-3)$ & - & $30(100)$ \\
7 anos & - & - & $38(100)$ \\
8 anos & - & $2(6,1)$ & $30(90,9)$ \\
\hline 9 anos & $1(3)$ & $3(10)$ & $27(90)$ \\
10 anos & - & $2(4,5)$ & $42(95,5)$ \\
11 anos & - & $3(7,9)$ & $35(92,1)$ \\
\hline 12 anos & - & - & $38(100)$ \\
\hline 13 anos & - & & \\
14 anos & & & \\
\hline & - & & \\
\hline
\end{tabular}

Conexões: revista da Faculdade de Educação Física da UNICAMP, Campinas, v. 12, n. 1, p. 126-141, jan./mar. 2014. ISSN: 1983-9030 


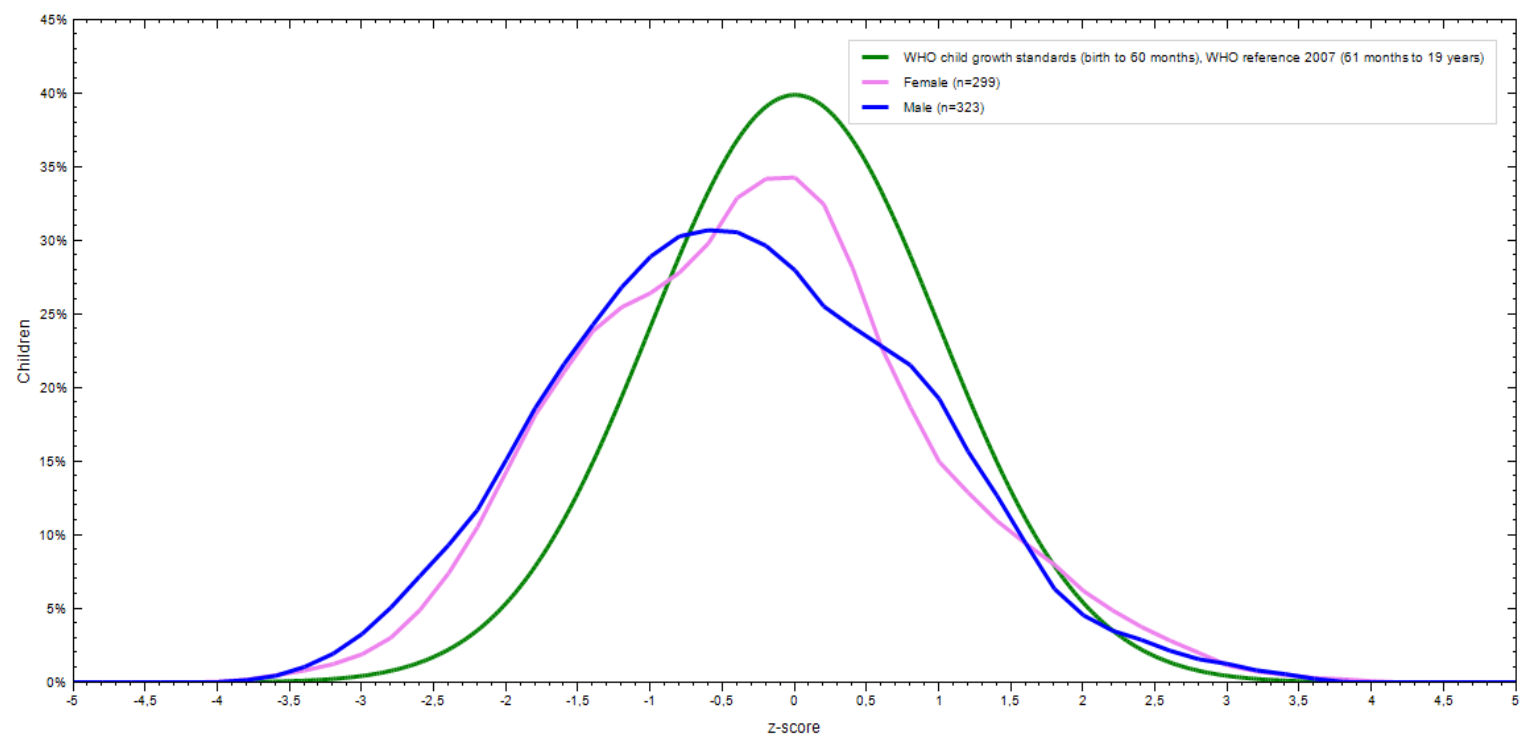

Figura 2: Curvas de Estatura para idade das crianças investigadas e o padrão da WHO (2007)

Os resultados referentes a estatura para a idade do presente estudo tem sido diferentes aos encontrados na região sul do Brasil onde a estatura dos indivíduos pesquisados obedeceu uma distribuição normal tanto com relação as diferentes faixas etárias como em ambos os sexo. ${ }^{1,3,5,17}$ Contudo, em conformidade com este estudo, pesquisas ${ }^{11,15,18,21}$ com crianças e adolescentes nordestinos revelaram um déficit de baixa estatura, sendo que esta prevalência em boa parte dos estudos foi superior ao esperado.

Os resultados referentes à classificação do IMC para idade de acordo com os estratos etários e sexo podem ser observados na Tabela 4 abaixo. Verificou-se novamente conforme o esperado, que a maioria dos indivíduos independente da idade indicou IMC adequado. Porém, verificou-se um percentual significativamente elevado de indivíduos, tanto no grupo masculino como feminino, com classificação do estado nutricional alusivo à magreza e magreza acentuada. Já com relação ao risco de obesidade os 
maiores valores encontrados estão entre os meninos de 09 anos (19\%) e as adolescentes de 14 anos $(15,4 \%)$. Quanto ao índice de obesidade, apenas os meninos de 08 anos $(7,9 \%)$ e as meninas de 06 e 08 anos $(10,7 \%)$ e $(10 \%)$ respectivamente, apontaram maiores valores percentuais para esta classificação. As curvas de IMC para idade dos grupos investigados novamente se diferem ligeiramente do padrão da $\mathrm{WHO}^{14}$, pois se observou uma projeção da área da curva para a esquerda justamente na área que determina o intervalo os escores $\mathrm{Z}$ de magreza e magreza acentuada referenciada a idade cronológica (Figura 3).

Tabela 4 - Classificação do IMC de acordo com os estratos etários e sexo

\begin{tabular}{cccccc}
\hline $\begin{array}{c}\text { Sexo e } \\
\text { Idade }\end{array}$ & $\begin{array}{c}\text { Magreza } \\
\text { acentuada } \\
\mathbf{f}(\boldsymbol{\%})\end{array}$ & $\begin{array}{c}\text { Magreza } \\
\mathbf{f}(\boldsymbol{\%})\end{array}$ & $\begin{array}{c}\text { Normal } \\
\mathbf{f}(\boldsymbol{\%})\end{array}$ & $\begin{array}{c}\text { Risco de } \\
\text { Obesidade } \\
\mathbf{f}(\%)\end{array}$ & $\begin{array}{c}\text { Obesidade } \\
\mathbf{f}(\boldsymbol{\%})\end{array}$ \\
\hline $\begin{array}{c}\text { Masculino } \\
6 \text { anos }\end{array}$ & $2(5,4)$ & $2(5,4)$ & $28(75,7)$ & $3(8,1)$ & $2(5,4)$ \\
7 anos & $1(2,8)$ & $2(5,6)$ & $30(83,3)$ & $2(5,6)$ & $1(2,8)$ \\
8 anos & $1(2,6)$ & $3(7,9)$ & $30(78,9)$ & $1(2,6)$ & $3(7,9)$ \\
9 anos & $2(9,5)$ & $1(4,8)$ & $14(66,7)$ & $4(19,0)$ & - \\
10 anos & $1(2,6)$ & $2(5,1)$ & $32(82,1)$ & $2(5,1)$ & $2(5,1)$ \\
\hline 11 anos & $1(2,3)$ & $3(7,0)$ & $34(79,1)$ & $4(9,3)$ & $1(2,3)$ \\
12 anos & $1(2,7)$ & $3(8,1)$ & $28(75,7)$ & $3(8,1)$ & $2(5,4)$ \\
\hline 13 anos & $2(5,1)$ & $2(5,1)$ & $30(76,9)$ & $3(7,7)$ & $2(5,1)$ \\
\hline 14 anos & - & $6(18,2)$ & $26(78,8)$ & $1(3,0)$ & - \\
\hline Feminino & & & & & \\
6 anos & - & $1(3,6)$ & $21(75,0)$ & $3(10,7)$ & $3(10,7)$ \\
\hline 7 anos & - & $2(6,1)$ & $28(84,8)$ & $2(6,1)$ & $1(3,0)$ \\
\hline 8 anos & - & $1(3,3)$ & $22(73,3)$ & $4(13,3)$ & $3(10,0)$ \\
\hline 9 anos & $1(2,6)$ & $3(7,9)$ & $29(76,3)$ & $3(7,9)$ & $2(5,3)$ \\
\hline 10 anos & - & $6(18,2)$ & $21(63,6)$ & $4(12,1)$ & $2(6,1)$ \\
\hline 11 anos & $1(3,3)$ & $2(6,7)$ & $23(76,7)$ & $3(10,0)$ & $1(3,3)$ \\
\hline 12 anos & - & $2(4,5)$ & $36(81,8)$ & $5(11,4)$ & $1(2,3)$ \\
\hline 13 anos & $2(5,3)$ & $1(2,6)$ & $33(86,8)$ & $1(2,6)$ & $1(2,6)$ \\
\hline 14 anos & $1(3,8)$ & $1(3,8)$ & $20(76,9)$ & $4(15,4)$ & - \\
\hline
\end{tabular}




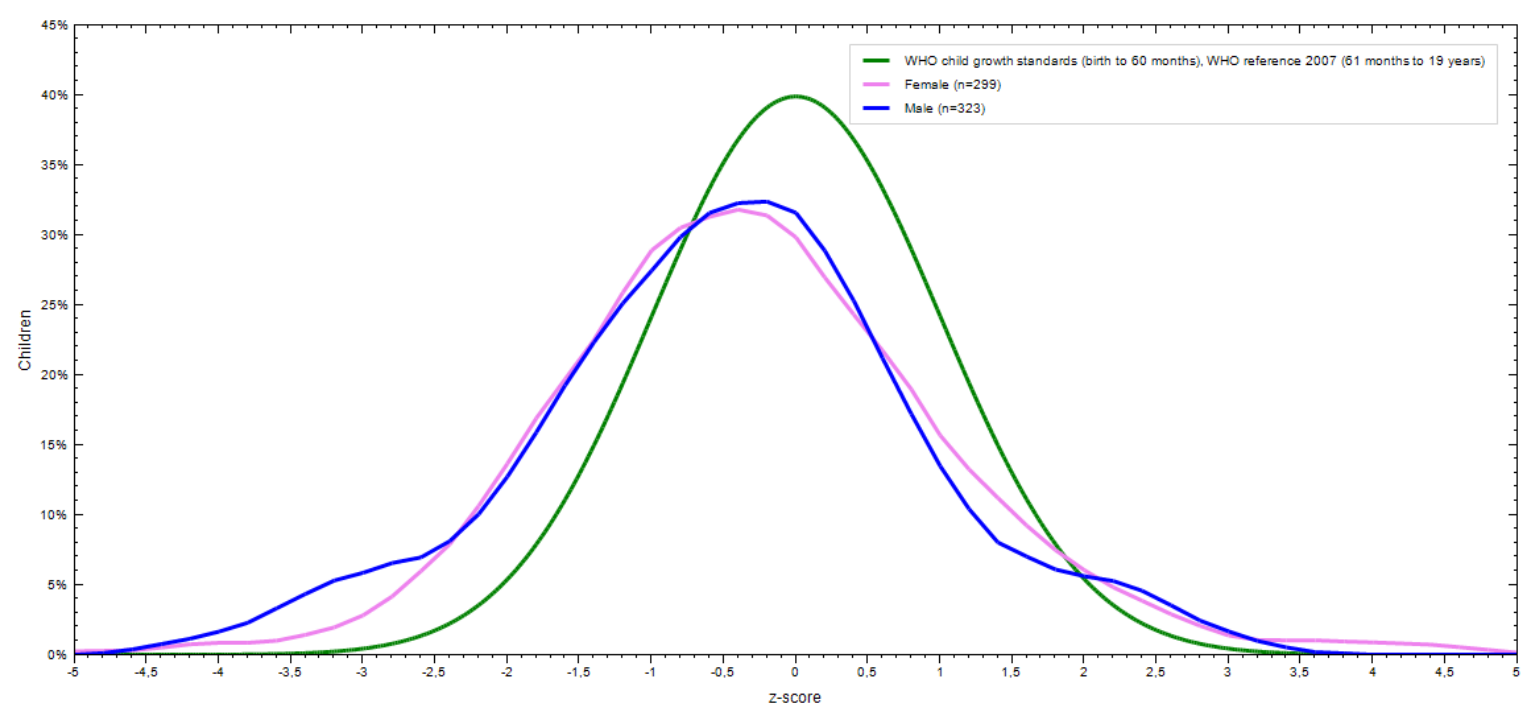

Figura 3: Curvas de IMC para idade das crianças investigadas e o padrão da WHO (2007)

Em relação ao estado nutricional novamente os resultados são divergentes aos encontrados em estudos prévios. ${ }^{19}$ Pesquisas realizados no sudeste e sul ${ }^{19}$ encontraram alto percentual de obesidade. Resultados de pesquisas ${ }^{15,18}$ publicadas no nordeste tem indicado, em conformidade com este estudo, um alto percentual de magreza e desnutrição. Em um estudo realizado em Sergipe constatou-se um percentual elevado de desnutrição principalmente nas meninas. ${ }^{21}$ Em uma região mais próxima ao norte do Ceará, um estudo também observou resultados similares em que percentuais significativos de crianças com magreza e magreza acentuada foram encontrados. ${ }^{11}$ 


\section{CONCLUSÃO}

A partir dos resultados encontrados neste estudo pode-se concluir que conforme o esperado a maioria dos indivíduos investigados independente da idade indicou estatura adequada percebeu-se também que não há uma diferença considerável entre meninas e meninos no referido indicador, entretanto verificou-se um percentual elevado de baixa estatura para a idade, sobretudo no grupo masculino, confirmados ao lançar os dados nas curvas de estatura para idade que revelaram uma inclinação para os escores de classificação que indicam baixa estatura. Estes achados revelam índices de crescimento estatural um pouco abaixo do esperado levando em consideração o padrão adotado neste estudo.

Quanto aos resultados do IMC verificou-se novamente conforme o esperado, que a maioria dos indivíduos investigados independente da idade indicou IMC adequado. Todavia, verificou-se um percentual significativamente elevado de indivíduos, tanto no grupo masculino, como feminino com classificação do estado nutricional alusivo à magreza e magreza acentuada, também confirmada ao lançar os dados nas curvas de IMC para idade.

Estes resultados podem indicar que estes indivíduos possam estar sofrendo uma carência nutricional consequentemente acarretando possíveis prejuízos no desenvolvimento psicomotor além de menor desempenho escolar.

Este fato reforça a importância de avaliações do crescimento físico e estado nutricional no âmbito escolar, principalmente no interior do Nordeste brasileiro, pois se trata de uma importante ferramenta para a compreensão da situação nutricional e estatural de uma determinada população, facilitando a formulação de políticas públicas que possam suprir determinadas carências de nutrientes ou excesso de gorduras promovendo uma ISSN: 1983-9030 
melhor qualidade na alimentação, assim como, estabeleçam atitudes de intervenção no sentido de prevenir distúrbios como o excesso de peso e desnutrição.

\section{REFERÊNCIAS}

${ }^{1}$ GLANER, M. F. Crescimento físico em adolescentes do norte gaúcho e oeste catarinense. Revista Brasileira de Ciência e Movimento, Brasília, v. 13, n. 2, p. 15-26, 2005.

${ }^{2}$ MENEZES, R. C. E.; OSÓRIO, M. M. Consumo energético-protéico e estado nutricional de crianças menores de cinco anos, no estado de Pernambuco, Brasil. Revista de Nutrição, Campinas. v. 20, n. 4, p. 337-347, jul./ago. 2007.

${ }^{3}$ DINIZ, I. M. S.; LOPES, A. S.; BORGATTO, A. F. Crescimento físico e composição corporal de escolares de diferentes grupos étnicos do estado do Rio Grande do Sul, Brasil. Revista Brasileira de Cineantropometria e Desempenho Humano, Florianópolis, v. 10, n. 1, p. 12-18, 2008.

${ }^{4}$ NHANTUBO, L. et al. Atividade física em crianças e jovens residentes em uma comunidade rural moçambicana: efeitos da idade, sexo e estado nutricional. Revista Panamericana de Salud Publica, Washington, v. 23, n. 3, p. 171-78, 2008.

${ }^{5}$ SALOMONS, E.; RECH, C. R.; LOCH, M. R. Estado nutricional de escolares de seis a dez anos de idade da rede municipal de ensino de Arapoti, Paraná. Revista Brasileira de Cineantropometria e Desempenho Humano, Florianópolis, v. 9, n. 3, p. 244-249, 2007.

${ }^{6}$ MONTEIRO, C. A. et al. Causas do declínio da desnutrição infantil no Brasil, 19962007. Revista Saúde Pública, Rio de Janeiro, v. 43, n. 1, p. 244-249, 2009. 
${ }^{7}$ NASCIMENTO, A. P. B.; FERREIRA, M. L.; MOLINA, S. M. G. Avaliação antropométrica de escolares em Piracicaba SP: da desnutrição para a obesidade. ConScientiae Saúde, São Paulo, v. 9, n. 4, p. 707-13, 2010.

${ }^{8}$ DELWING, K. B. B.; REMPEL, C.; BOSCO, S. M. D. Prevalência de sobrepeso e obesidade em escolares entre 6 e 11 anos de um município do interior do RS. ConScientiae Saúde, São Paulo, v. 9, n. 2, p. 173-178, 2010.

${ }^{9}$ ABRAnTES, M. M.; LAMOUnIER, J. A.; COLOSIMO, E. A. Prevalência de sobrepeso e obesidade em crianças e adolescentes das regiões Sudeste e Nordeste. Jornal de Pediatria, Rio de Janeiro, v. 78, n. 4, p. 335-40, 2002.

${ }^{10}$ BALABAN, G.; SILVA, G. A. P. Prevalência de sobrepeso e obesidade em crianças e adolescentes de uma escola da rede privada de Recife. Jornal de Pediatria, Rio de Janeiro, v. 77, n. 2, p. 96-100, 2001.

${ }^{11}$ NOBRE, G. C. et al. Índices de estatura para idade e IMC para idade em escolares de um município do nordeste brasileiro. In: CONGRESSO NORTE E NORDESTE DE PESQUISA E INOVAÇÃO, 7., 2012, Palmas. Anais... v. 1, p. 245-49, 2012.

${ }^{12}$ GAYA, A. C. Ciências do movimento humano: introdução a metodologia de pesquisa. Porto Alegre: Artmed, 2008.

${ }^{13}$ FEDERAÇÃO DAS INDÚSTRIAS DO ESTADO DO RIO DE JANEIRO (FIRJAN). Índice FIRJAN de desenvolvimento municipal. Município Acopiara - CE (Ano base 2010): IFDM. Disponível em: <http://www.firjan.org.br/ifdm>. Acesso em: 23 fev. 2012.

${ }^{14}$ WORLD HEALTH ORGANIZATION (WHO). Child growth standards: length/height-for-age, weight-forage, weight-for-length, weight-for-height and body mass index-for-age. Geneva, 2006. Disponível em: http :< //www.who.int/childgrowth/en/>. Acesso em: $12 \mathrm{dez} 2013$.

Conexões: revista da Faculdade de Educação Física da UNICAMP, Campinas, v. 12, n. 1, p. 126-141, jan./mar. 2014. ISSN: 1983-9030 
${ }^{15}$ SILVA, S. P. Estudo longitudinal-misto do crescimento e desempenho motor em crianças e adolescentes da região do Cariri Cearense, Brasil. 2010. 238f. Tese (Doutorado em Ciências do Desporto) - Faculdade de Desporto, Universidade do Porto, Porto, 2010.

${ }^{16}$ GUEDES, D. P.; MENDES, R. R. Crescimento físico e estado nutricional de escolares do Vale do Jequitinhonha, Minas Gerais, Brasil. Revista Brasileira de Cineantropometria e Desempenho Humano, Florianópolis, v. 14, n. 4, 2012.

${ }^{17}$ AMORIM, S. T. S. P.; RODRIGUES, A. G. M.; STOLARSKI, M. C. Estatura de adolescentes matriculados em escolas da rede pública no estado do Paraná, Brasil. Revista de Nutrição, Campinas. v. 22, n. 2, p. 195-205, mar./abr. 2009.

${ }^{18}$ ALVES, J. G.; SIQUEIRA, P. P.; FIGUEIROA, J. N. Overweight and physical inactivity in children living in favelas $s$ in the metropolitan region of Recife, Brazil. Jornal de Pediatria, Rio de Janeiro, v. 85, v. 2, p. 67-71, 2009.

${ }^{19}$ VANZELLI, A. S. et al. Prevalência de sobrepeso e obesidade em escolares da rede pública do município de Jundiaí, São Paulo. Revista Paulista de Pediatria, São Paulo, v. 26, n. 1, p. $48-53,2008$.

${ }^{20}$ JANUÁRIO, R. S. B. et al. Índice de massa corporal e dobras cutâneas como indicadores de obesidade em escolares de 8 a 10 anos. Revista Brasileira de Cineantropometria e Desempenho Humano, Florianópolis, v. 10, n. 3, p. 266-270, 2008.

${ }^{21}$ PETROSKI, E. L.; SILVA, R. J. S.; PELEGRINI, A. Crescimento físico e estado nutricional de crianças e adolescentes da região de Cotinguiba, Sergipe. Revista Paulista de Pediatria, São Paulo, v. 26, n. 3, 2008.

Recebido em: 08 fev. 2014 Aceito em: 25 fev. 2014 Contato: Glauber Carvalho Nobre glauber_nobre@hotmail.com

Conexões: revista da Faculdade de Educação Física da UNICAMP, Campinas, v. 12, n. 1, p. 126-141, jan./mar. 2014. ISSN: 1983-9030 